\title{
Molecular pathogenesis and clinical management of Fanconi anemia
}

\author{
Younghoon $\mathrm{Kee}^{1}$ and Alan D. D'Andrea ${ }^{2}$ \\ 1Department of Cell Biology, Microbiology, and Molecular Biology, University of South Florida, Tampa, Florida, USA. \\ ${ }^{2}$ Department of Radiation Oncology and Pediatrics, Dana-Farber Cancer Institute, Harvard Medical School, Boston, Massachusetts, USA.
}

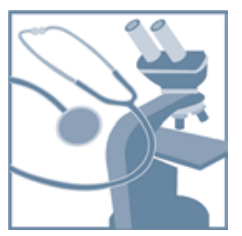

Fanconi anemia (FA) is a rare genetic disorder associated with a high frequency of hematological abnormalities and congenital anomalies. Based on multilateral efforts from basic scientists and clinicians, significant advances in our knowledge of FA have been made in recent years. Here we review the clinical features, the diagnostic criteria, and the current and future therapies of FA and describe the current understanding of the molecular basis of the disease.

\section{Diagnosis of FA}

Fanconi anemia (FA) is a genetic disorder characterized by multiple congenital anomalies and hematological abnormalities and predisposition to a variety of cancers (Figure 1). The classic diagnostic test for FA is the assessment of cellular hypersensitivity to DNA interstrand crosslinking agents (ICLs), such as diepoxybutane (DEB) and mitomycin C (MMC) $(1,2)$. Exposure of FA cells to these agents results in high levels of chromosomal aberrations, particularly chromosomal breaks and radial formations. The DEB-induced chromosome breakage assay (DEB test) has been widely used for the primary diagnosis of FA. Due to possible somatic mosaicism (i.e., the presence of two or more cell populations with different genotypes), measuring the percentage of cells with aberrations is more informative than measuring the number of aberrations per cell. The chromosome breakage test yields occasional false positives, as other genetic disorders, such as Nijmegen breakage syndrome and Roberts syndrome, also display aberrant chromosomes upon exposure to these DNA ICLs (3).

Examining the cell cycle profile of peripheral blood lymphocytes is also useful in diagnosing FA. FA cells display a marked increase of cells in G2/M phase (4N DNA content), either before or after treatment with DNA ICLs. However, the definitive diagnostic test is the complementation test, also known as FA subtyping. In this test, patient-derived FA cells are transduced with retroviruses that carry cDNAs complementing the different FA subtypes. Transduction with the appropriate FA complementation group (FANC) cDNA will correct the cellular FA phenotypes, such as the chromosomal aberrations and hypersensitivity to DNA ICLs. In addition, clinical manifestations such as short stature, skeletal deformities, and café-au-lait spots on skin can aid diagnosis (4). An early diagnosis of FA is important for clinical management, particularly because FA patients are predisposed to multiple malignancies (see below).

\section{Pathophysiology of FA}

Individuals with FA display several congenital defects, but approximately $25 \%-40 \%$ of FA patients are physically normal (1). Approximately half of children with FA have congenital skeletal

Conflict of interest: The authors have declared that no conflict of interest exists. Citation for this article: JClin Invest. 2012;122(11):3799-3806. doi:10.1172/JCI58321. anomalies, frequently of the thumb and forearm. The thumbs are usually smaller (hypoplastic), duplicated, or absent. The radius of the forearm may also be smaller or absent (5). Many FA individuals display endocrine abnormalities. Approximately half of FA individuals have short stature, correlating with insufficient growth hormone production and hyperthyroidism. Some FA individuals have normal stature and do not have an obvious deficiency in growth hormone production. Additionally, abnormal glucose or insulin metabolism is associated with FA. As opposed to reduced insulin in diabetes, FA individuals usually have a higher level of serum insulin. Approximately $8 \%$ of individuals with FA are reported to be diabetic, while up to $72 \%$ showed elevated insulin $(6,7)$. In addition, osteoporosis is associated with FA (6-9).

Hematologic abnormalities represent the most prevalent pathologic manifestation of FA. Approximately $75 \%-90 \%$ of FA patients develop bone marrow failure, ranging from mild to severe, during the first decade of life $(10,11)$. In addition, most FA individuals develop varying degrees of blood disease, including aplastic anemia, myelodysplastic syndrome (MDS), or acute myeloid leukemia (AML). The risk of AML occurrence is approximately 800-fold higher than that of the general population, with a median age of onset of 14 years. Recent reports revealed a common pattern of specific chromosomal abnormalities in FA patients with MDS or AML (e.g., gain of 1q23-32, 3q26), which suggests that these abnormalities can be useful predictive markers (4, 12-14). The exact cause of these hematopoietic defects is unclear, although increasing evidence suggests an underlying intolerance of FA hematopoietic cells to oxidative stress (15).

Although FA is mainly a pediatric disease, adult FA patients ( $>18$ years of age) now represent an increasing proportion of the FA patient population due to improved management of young FA patients and more rigorous diagnostic testing in adults. A major health threat faced by adult FA patients is the risk of cancer (16). In addition to hematologic cancers, solid tumors, particularly squamous cell cancers (SCCs) of the head and neck and cervical/gynecological cancers, occur at markedly higher rates in FA patients (17). Approximately one-third of FA patients will develop a solid tumor by the fourth decade of life (18). The relative contribution of human papillomavirus (HPV) infection to SCC in FA patients is unknown, and published studies have yielded conflicting results (19-21). 


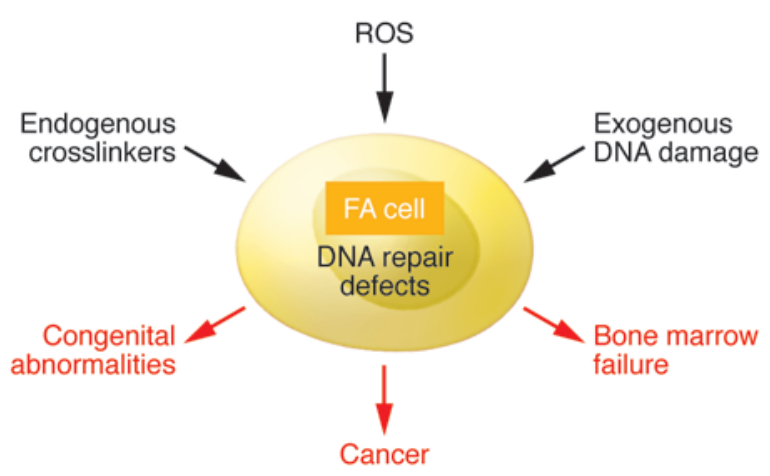

In addition to the hematological abnormalities and increased cancer susceptibility, FA individuals exhibit other clinical problems, such as hearing loss and ear anomalies as well as reduced fertility (5). Reduced sperm count is associated with male FA patients, and premature menopause is associated with female patients (22). The rate of successful pregnancy is approximately $15 \%$ among nontransplanted FA patients (22), although improved fertility and pregnancy after HSC transplantation (HCT) has been reported (23). Consistent with the reduced fertility in FA patients, some studies on knockout mouse models of Fanca, Fancc, Fancg, and Fancd 2 showed pronounced hypogonadism and impaired fertility, with females more severely affected than males $(24,25)$.

\section{Biology of FA}

FANCs. To date, 15 FANCs have been identified (FANCA, FANCB, FANCC, FANCD1, FANCD2, FANCE, FANCF, FANCG, FANCI, FANCJ, FANCL, FANCM, FANCN, FANCO, and FANCP; Table 1). The majority of FA genes are located on autosomes, with the exception of FANCB, which is on the X chromosome. FA patients with mutations in any of these FA genes share a characteristic clinical and cellular phenotype, and these 15 gene products

\section{Figure 1}

Pathophysiology of FA. FA cells are highly vulnerable to endogenous insults, such as ROS, other sources of DNA crosslinkers (e.g., formaldehyde), and exogenous DNA-damaging agents. Repeated exposure to these insults in FA cells during developmental stages can lead to high incidence of congenital abnormalities, bone marrow failure, and cancers.

\section{Table 1}

15 FA subtypes

\begin{tabular}{|c|c|c|c|c|c|}
\hline Gene (alias) & Locus & $\begin{array}{l}\text { Mutation } \\
\text { frequency }\end{array}$ & $\begin{array}{l}\text { Amino } \\
\text { acids }\end{array}$ & $\begin{array}{l}\text { Protein MW } \\
\quad(k D a)\end{array}$ & Notable protein function \\
\hline FANCA & $16 q 24.3$ & $\sim 66 \%$ & 1,455 & 163 & Scaffold for FA core complex \\
\hline FANCB & Xp22.31 & $\sim 2 \%$ & 859 & 95 & Scaffold for FA core complex \\
\hline FANCC & $9 q 22.3$ & $\sim 10 \%$ & 558 & 63 & Scaffold for FA core complex \\
\hline FANCD1 (BRCA2) ${ }^{\mathrm{A}}$ & $13 q 12.3$ & $\sim 2 \%$ & 3,418 & 380 & Recruits RAD51 and promotes HR repair \\
\hline FANCD2 & $3 p 25.3$ & $\sim 3 \%$ & 1,451 & 162 & Monoubiquitinated. Recruits FAN1, FANCP to chromatin \\
\hline FANCE & $6 \mathrm{p} 21.3$ & $\sim 2 \%$ & 536 & 60 & Scaffold for FA core complex. Probable adaptor for FANCD2 \\
\hline FANCF & $11 p 15$ & $\sim 2 \%$ & 374 & 42 & Scaffold for FA core complex \\
\hline FANCG $(X R C C 9)$ & $9 p 13$ & $\sim 10 \%$ & 622 & 70 & Scaffold for FA core complex \\
\hline $\mathrm{FANCl}$ & $15 q 25-26$ & $<2 \%$ & 1,328 & 150 & Monoubiquitinated. Forms heterodimer with FANCD2 \\
\hline FANCJ $(B A C H 1, B R I P 1)^{A}$ & $17 q 22.3$ & $<2 \%$ & 1,249 & 150 & Interacts with BRCA1. DNA helicase, ATPase \\
\hline FANCL $($ PHFG) & $2 \mathrm{p} 16.1$ & Rare & 375 & 43 & Recruits UBE2T via PHD E3 domain. Monoubiquitinates FANCD2 and FANCI \\
\hline FANCM & $14 \mathrm{q} 21.3$ & Rare & 2,014 & 250 & $\begin{array}{l}\text { Scaffold for FA core complex. Branch migration/ATPase activity. } \\
\text { Interacts with BLM }\end{array}$ \\
\hline FANCN (PALB2) ${ }^{A}$ & $16 p 12.1$ & Rare & 1,186 & 130 & Mediates interaction between BRCA1 and BRCA2 during HR \\
\hline FANCO $(\text { RAD51C })^{\mathrm{A}}$ & $17 q 22$ & Rare & 376 & 43 & Promotes HR. Interacts with RAD51 and RAD51 homologs \\
\hline FANCP $(S L X 4)^{\mathrm{B}}$ & $16 p 13.3$ & Rare & 1,834 & 200 & $\begin{array}{l}\text { Functions as holiday junction resolvase with SLX1. } \\
\text { Interacts with XPF-ERCC1 and EME1-MUS81 nucleases }\end{array}$ \\
\hline
\end{tabular}

AFamilial susceptibility to breast cancer. ${ }^{B}$ Familial breast cancer susceptibility unclear (minor, if any). 


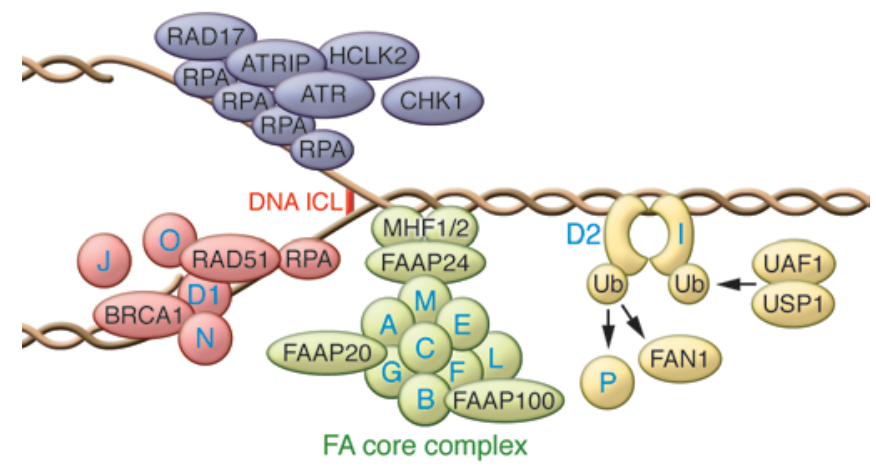

\section{Figure 2}

FA pathway. ATR-mediated signal activates the FA pathway at the stalled replication forks, leading to the activation of the FA core complex and monoubiquitination of the FANCD2/FANCI heterodimer. ATR phosphorylates multiple FA proteins for activation. Monoubiquitinated D2/I recruits FAN1 nuclease and FANCP (which associates with several nucleases), which might participate in nucleolytic incision near crosslinked DNA. D2/I might recruit TLS polymerases (not shown) and HR factors, including FANCD1 (BRCA2) and RAD51. FANCO (RAD51C) is known to participate in the HR step. The USP1/UAF1 complex deubiquitinates D2/I to complete the pathway. 15 FA proteins (blue) are shown. Other important factors that crosstalk with the FA pathway, such as PCNA, $\gamma-\mathrm{H} 2 \mathrm{AX}$, TOPBP1, MSH2, TLS polymerases, and BLM complexes, are not shown for simplicity. A DNA ICL (red) is illustrated between the DNA double strand. intervention, such as unrelated donor bone marrow transplantation. Patients with FANCD1 have earlier onset and increased incidence of leukemia and solid tumors (35). In general, early diagnosis and identification of FANC mutations is critical for the informed genetic counseling of parents of FA patients with regard to future pregnancies.

Role of the FA pathway in DNA repair during $S$ phase. DNA ICLs are highly toxic lesions that block essential DNA metabolism, such as DNA replication and transcription. Hypersensitivity to ICL in FA is associated with the accumulation of G2/M-arrested cells. The cell cycle arrest at $\mathrm{G} 2 / \mathrm{M}$ is due to increased activity of checkpoint kinase CHK1, and depleting CHK1 suppresses the G2/M arrest phenotypes of FA cells (36). These observations suggest that FA cells are inherently defective in repairing DNA damage. Indeed, increasing evidence affirms that the FA proteins are directly involved in repairing damaged DNA (26).

Among the 15 FA gene products identified so far, the eight upstream FA proteins (FANCA, FANCB, FANCC, FANCE, FANCF, FANCG, FANCL, and FANCM) form a nuclear complex, termed the FA core complex, which possesses intrinsic E3 ubiquitin ligase activity (Figure 2). Most FA proteins in the FA core complex do not contain any recognizable enzymatic domain, which suggests that their primary function is a scaffold (Table 1). The key domain in the complex is the PHD-type RING finger domain at the $\mathrm{C}$ terminus of FANCL. The PHD domain interacts with an E2 ubiquitin-conjugating enzyme, UBE2T, depletion of which in cultured cells leads to a characteristic FA phenotype (37). The primary function of the FA core complex is to monoubiquitinate two FA proteins, FANCD2 and FANCI, which are expressed as a constitutive heterodimer $(38,39)$. A crystal structure of the FANCD2/FANCI heterodimer (also referred to as the ID complex) suggests that this complex can recognize various DNA structures that may arise from stalled replication forks (40); however, how monoubiquitylation affects the activity or conformation of this heterodimer is unknown. Monoubiquitylated FANCD2 serves as a signal for recruiting downstream effector proteins that have affinity for the ubiquitin. Fanconi-associated nuclease 1 (FAN1) is recruited to the monoubiquitylated form of FANCD2 via its ubiquitin-binding UBZ domains (41-44) and is thought to participate in ICL repair through its nuclease activity.

The major DNA repair pathway regulated by the FA proteins is homologous recombination (HR), a process that repairs DNA double strand breaks (DSBs). DSBs are intermediate lesions during ICL repair, and HR is critical to their repair. Cells lacking FA proteins are deficient in promoting HR activities. Although the mechanism remains unclear, monoubiquitylated FANCD2/FANCI is thought to functionally associate with the downstream FA proteins critical for HR, including FANCD1 (i.e., BRCA2; 45, 46), FANCN (PALB2; ref. 47), and possibly FANCO (RAD51C) and FANCJ (BACH1; ref. 32). FANCD2 also colocalizes with key HR factors, such as BRCA1 and RAD51 (48). Although the upstream FA proteins are required for HR activity, the degree of the HR defect is milder compared with defects resulting from loss of the downstream FA proteins. FANCD2/FANCI proteins do not appear to directly regulate chromatin loading of the key HR enzyme RAD51 (49). However, RAD51 activity is directly regulated by FANCD1, FANCN, and FANCO, possibly accounting for the phenotypic differences. These downstream FA proteins are also potentially involved in a wider range of HR repair and not limited to ICL repair.

FA proteins may promote HR activity by different mechanisms. Recent reports suggest that FA proteins actively suppress nonhomologous end-joining (NHEJ), an error-prone DSB repair mechanism that functions during the G1 phase of the cell cycle $(50,51)$. Improper activation of NHEJ factors was observed in FA pathway-deficient cells, resulting in chromosomal rearrangements and aberrations during $\mathrm{S}$ phase. Several mechanisms are possible: (a) FA proteins may restrict the access of NHEJ factors, such as the Ku70-80 heterodimer, to the DSB ends during the ICL repair process; or (b) cryptic nuclease activity of FANCD2 may generate DNA structures at DSB ends recognized by HR factors $(1,27,51)$.

In addition to HR, FA proteins coordinate both nucleolytic incision and DNA translesion synthesis (TLS) during ICL repair. The current model suggests that replication fork stalling by ICL requires subsequent incision of the ICL flanking lesion and unhooking of the ICL by endonuclease activities $(26,52)$. Recruitment of endonucleases MUS81-EME1 or XPF-ERCC1 is likely involved in this step, although genetic studies indicate that XPF-ERCC1 may be the more relevant enzyme in the FA pathway $(53,54)$. In addition, FAN1 or SLX4-SLX1 nucleases may also be involved in this step. Incision of ICL is followed by TLS, a damage tolerance mechanism in which specialized low-fidelity polymerases bypass bulky damaged lesions. Cells with disrupted TLS polymerase (e.g., REV3) display FA-like phenotypes, such as hypersensitivity to DNA ICLs $(55,56)$. A biochemical study using the Xenopus replication system demonstrated that the monoubiquitylated FANCD2/FANCI complex is required for both nucleolytic incision and TLS steps during ICL repair (57). Although the incision step is not required for monoubiquitylation of FANCD2, it may be required for the chromatin recruitment of FANCD2 (58). Interest- 
ingly, SLX4, a scaffold protein that binds to MUS81-EME1, XPFERCC1, and SLX1 endonucleases, was recently identified as a the fifteenth FA subtype, FANCP (59-61). FANCP is not required for FANCD2 monoubiquitylation, but contains two UBZ domains that are required for recruitment to monoubiquitylated FANCD2 (62). Several additional FA-associated proteins are integral to the pathway. These include FA-associated protein $24 \mathrm{kDa}$ (FAAP24; ref. 63), FAAP100 (64), FANCM-associated histone fold protein 1 (MHF1), and MHF2 $(26,65)$, all of which are part of the FA core complex. The recently identified FAAP20 is also an integral part of the FA core complex (66-68). Cells rendered deficient in these FA-associated proteins display the classical FA phenotypes, including sensitivity to DNA ICLs and G2/M cell cycle arrest. However, FA patients with these genetic mutations have not been found, so these genes do not have FANC assignments.

In addition to these FA or FA-associated proteins, the FA pathway network broadly includes other regulatory proteins. Ubiquitin-specific peptidase 1 (USP1) and the USP1-associated protein (UAF1) regulate deubiquitination of FANCD2/FANCI and are required for completion of the FA pathway $(69,70)$. The ATR/ ATRIP heterodimer is required for sensing single-strand DNA generated during $S$ phase and for the induction of FANCD2/FANCI monoubiquitylation in response to DNA damage (71). Proteins involved in ATR signaling, such as RPA (single-strand DNA binding protein), RAD17, RAD9 (9-1-1 checkpoint complex), CHK1, and HCLK2, are required for the efficient induction of FANCD2/ FANCI monoubiquitylation $(36,72)$. FA proteins also crosstalk with MSH2/MSH3 mismatch proteins (73) and BLM helicase (74, $75)$ during ICL repair. Thus, defective DNA repair mechanisms during $S$ phase may render FA cells vulnerable to endogenous damage or oxidative stress.

Role of the FA pathway in cytokinesis. The primary DNA repair function of the FA pathway is exerted during $S$ phase. However, the role of FA proteins may extend to $\mathrm{M}$ phase, especially in cytokinesis. FA cells exhibit instability at chromosomal fragile sites, the regions in the mitotic chromosomes that serve as a marker for unrepaired DNA $(76,77)$. A series of recent studies suggested that the FA proteins provide a surveillance mechanism for monitoring unrepaired DNA during cytokinesis (78-80). Fragile sites were stained with FANCD2 and FANCI, and the absence of these FA proteins in these sites was associated with increased chromosome instability and binucleated cells (cells containing two nucleuses). These binucleated cells underwent apoptosis and are a potential cause of bone marrow failure in FA. The fragile sites are connected with mitotic DNA structures called ultrafine DNA bridges (UFBs). Replication errors during $S$ phase may increase UFB structures during $\mathrm{M}$ phase, possibly more so in FA cells, which may cause failure in cytokinesis and generation of binucleated cells. The normal function of the FA proteins at the fragile sites and UFB remains uncertain. Several other DNA repair proteins that are linked to the FA pathway, such as ATR, BRCA1, and RAD51, also regulate the stability of fragile sites, suggestive of a broader role of the FA-BRCA network during cytokinesis.

\section{Management of FA patients}

Treatment of bone marrow failure. Bone marrow failure in FA patients typically develops during the first decade of life (81). Androgen therapy is effective for treating bone marrow failure in some FA patients. Synthetic androgens, such as oxymetholon and danazol, have been effective in treating hematopoietic defects in FA patients
$(82,83)$, although long-term androgen use has side effects that include hirsutism and increased liver tumor incidence. Although androgens increase red blood cell counts and platelet counts (84), the exact mechanism of androgens in the hematopoietic system is unclear. Hematopoietic growth factors (e.g., G-CSF and GM-CSF) have been helpful in improving the neutrophil counts in FA (82).

HCT remains the primary treatment of choice for bone marrow failure in FA. Because of the underlying DNA repair defect in FA, extensive chemoradiation used in the transplantation procedure can be highly toxic. Histocompatible (matched) sibling donor transplant remains the best treatment for FA and gives the best outcome, if performed early (i.e., prior to the development of MDS or leukemia; refs. 81, 85). Still, transplant survivors experience multiple complications, including physical injury from chemoradiation (including pulmonary and renal toxicity and veno-occlusive disease), graft-versus host disease (GvHD), immune injury, sterility, and endocrinopathies. Optimization of the HCT conditioning regimen, such as use of lower-dose cyclophosphomide (alkylating agent), a fludarabine-based reduced-intensity regimen (immunosuppressive), or nonirradiation, continue to limit these complications while maintaining sufficient engraftment rates (85-89). As most FA patients do not have a histocompatible sibling, some families have turned to preimplantation genetic diagnosis (PGD) for generation of a suitable donor (90). HCT from alternate (unrelated or HLA mismatched) donors is associated with a higher risk of complications and a lower survival rate. However, the survival rate of alternate-donor HCT has improved steadily since Gluckman and colleagues initially reported the outcome of alternate-donor HCT for FA patients in 1995 (91). More recently, reduction of the irradiation in the conditioning regimen has limited the toxic effects and improved the survival rates of alternate-donor HCT (89). While HCT is highly successful in extending the life expectancy of FA patients, managing the long-term complications of HCT now has greater importance. Additionally, HCT does not treat nonhematopoietic complications of FA, such as increased cancer susceptibility.

Gene therapy. There has been a resurgence of interest in gene therapy for FA. In many ways, FA is an ideal candidate disease for gene therapy. Subtyping FA patients is relatively straightforward, allowing identification of the mutant FA gene and generation of retroviral or lentiviral vectors carrying the wild-type cDNA to complement cells. Transduced and cDNA-complemented cells should have a clear selective advantage in vivo over endogenous marrow cells. Furthermore, gene therapy provides a method for generation of autologous donor cells. However, successful gene therapy for FA has been delayed by several technical obstacles. First, FA patients lack sufficient HSCs for ex vivo transduction. Accordingly, marrow should be collected and stored early in the life of the FA patient. New methods are needed to expand FA bone marrow cells ex vivo, which could potentially be developed with the use of HOXB4 and DELTA-1 (92). Second, improvements in the efficien$c y$ of viral transduction are required. The benefit of reducing oxidative stress, combined with shorter ex vivo transduction periods, has been shown for improving gene transduction efficiency in FA HSCs and may hold promise for enhancing FA gene therapy (93). Finally, gene therapy continues to pose a risk of leukemia, driven by the integration of the therapeutic vector near a proto-oncogene, followed by the expansion of a malignant clone. Improvements in conditioning regimens and in the generation of self-inactivating retroviral vectors and lentiviral vectors may further improve the efficiency and safety of gene therapy for FA. 


\section{Table 2}

Potential future therapies for FA

\section{Therapeutic approach}

Improved gene therapy

Antioxidative compounds

Small molecule inhibitors

(e.g., inhibitors of CHK1,

DNA-PK, p38 MAP kinase)

Ex vivo graft of revertant HSCs

iPS

\section{Rationale}

Improving sufficient isolation of FA HSCs through genetic manipulations. Difficulty in finding matched sibling donors for HCT, and the effects observed in somatic mosaic patients, wherein a small number of revertant cells are able to reconstitute hematopoietic counts

ROS is a significant source of endogenous DNA damage, particularly for FA. Reducing ROS rescues FA phenotypes in mouse models and human cell cultures

Suppressing CHK1 confers clinical improvements in some FA patients. Inhibiting NHEJ pathway suppresses FA phenotypes in vitro cell cultures and $C$. elegans model. Inhibiting MAP kinase suppress TNF- $\alpha$ sensitivity of FA cells in vitro

Spontaneous (natural) correction of mutant FA genes is associated with improved clinical manifestations. The small number of revertant HSCs can potentially be isolated and expanded in vitro, which will circumvent ectopic gene correction

Difficulty of finding matched sibling donors of HCT and difficulty of isolating sufficient amount of HSCs for ex vivo genetic manipulation can be overcome by iPS technology

\section{Potential limitations}

Potential insertional mutagenesis and malignant outgrowth of mutant clones. Questionable long-term ability of graft cells to maintain hematopoiesis

Excessive usage of antioxidants may cause DNA damage-induced apoptosis

Long-term use of the inhibitors may enhance tumorigenesis or immune suppression

Long-term ability of the graft cells for hematopoietic functionality questionable

Oncogenic potential of virus-mediated gene insertion can be problematic. Reprogramming efficiency must be improved for clinical applications
Treatment for endocrine dysfunction. Hypothyroidism is observed for many FA patients. One study found that thyroid treatment in FA children with short stature for 7 months led to significant growth improvement, suggesting that FA children with short stature may benefit from thyroid treatment (94). Approximately 50\%-70\% of FA children have growth hormone deficiency, and extra growth hormone is usually given for these individuals $(94,95)$. Whether there is an increased risk of AML or other cancers in response to longterm growth hormone treatment remains controversial.

Management of cancers. FA patients have a high frequency of AML and SCCs of the head and neck and gynecologic system $(16,96)$. With the prolonged survival after successful transplantation, treating and managing FA-related cancers remains an important challenge. Due to the cytotoxicity of chemo- and radiotherapeutic regimens for FA patients, the treatment of FA-related cancers must differ from the treatment strategy for non-FA individuals. Prevention and close surveillance of cancer occurrence are critical elements of FA patient clinical management. FA patients must have annual bone marrow aspirates to rule out premalignant clonal expansion and frequent dental evaluations to rule out early oral cavity SCCs. Women with FA require frequent gynecologic exams to identify early SCCs, and all FA patients should receive HPV vaccinations. Some FA subtype-specific patterns of cancer exist. For instance, patients with subtype D1 have earlier onset and increased incidence of leukemia and solid tumors. FANCD1 mutations are associated with a variety of tumors, including Wilms tumor, neuroblastoma, and brain tumors such as medulloblastoma $(35,97,98)$. Moreover, heterozygote carriers from FA subtype D1, J, N, and O families may be predisposed to breast, ovarian, and pancreatic cancers.

\section{Future of FA therapy}

Despite a greater understanding of the biology of FA, the mechanisms underlying the hematopoietic defects, developmental defects, and bone marrow failure of FA remain elusive. The defective DNA repair capacity of FA stem cells may underlie their hyper- sensitivity to endogenous DNA damage, such as damage from $\operatorname{ROS}(15,99)$ or from serum formaldehyde $(100,101)$. Such hypersensitivity may lead to decreased hematopoietic cellularity (stem cell loss in early development), similar to phenotypes observed in ATM-deficient or ligase IV-deficient (LIG4-deficient) mice (102, 103). Therefore, limiting the potential sources of endogenous DNA-damaging agents may protect HSCs in FA. A recent study showed that resveratrol, an activator of SIRT1 with antioxidant effect, partially rescues the hematopoietic defects of Fancd2 $2^{-/-}$mice (104), which suggests that increased sensitivity to ROS is a contributing factor in FA. Antioxidants may also delay tumor onset in FA; the antioxidizing small molecule Tempol was shown to delay tumor formation in a Fancd2 $2^{-/-} \operatorname{Tr} p 53^{+/-}$mouse model (105). Screening of small molecules or natural compounds that rescue the hematopoietic defects in FA, particularly those that reduce oxidative DNA damage, may hold promise for future FA therapies.

Several reports provide evidence of somatic reversion in FA, leading to mosaicism. Mosaicism results from spontaneous reverse mutations that restore functional FA alleles (106-109). Reverse mosaicism in FA can provide a selective advantage, and the corrected HSCs can improve clinical outcome. Reverse mosaicisim, often referred to as natural gene therapy, suggests that replenishing even a limited fraction of corrected HSCs can significantly improve the hematopoietic defects in FA. The revertant FA stem cells could, in principle, be isolated, expanded in vitro, and grafted back to the patient as an autologous transplant (Table 2). Successful attempts have been made to transplant revertant keratinocytes as a treatment for skin disorders $(110,111)$. However, questions remain about the ability of corrected cells to sustain the hematopoietic functionality in the long term. Furthermore, the nonrevertant FA cells are still prone to the development of a malignant clonal abnormality (112). Spontaneous mutations in non-FA genes might confer a selective growth advantage to the corrected clones in FA. Individuals with FA were identified by acquired clonal selection of 
cells with downregulated ATR-CHK1 proteins and abrogated G2/M checkpoint (113). These individuals had mild bone marrow abnormalities and lived to adulthood, which suggests that G2/M checkpoint abrogation has clinical benefits for FA cells. Long-term effectiveness is questionable, however, as some of these patients eventually develop leukemia or myelodysplasia.

The bone marrow failure in FA individuals may result from hyperactive checkpoint responses in hematopoietic stem and progenitor cells (HSPCs). Primary bone marrow cells from FA patients have elevated levels of $\mathrm{p} 53$ and of its downstream effector protein, p21 (A.D. D'Andrea, unpublished observations). Elevated p53 and p21 appear to account, at least in part, for the enhanced cell cycle arrest and apoptosis of HSPCs in the bone marrow of FA patients. A similar mechanism of p53-mediated bone marrow apoptosis has been observed in another inherited bone marrow failure syndrome, Diamond-Blackfan anemia (114). Small molecule inhibitors of p53 may, in principle, rescue this HSPC apoptosis and improve hematopoiesis in FA patients, but at the risk of increasing leukemia incidence.

Finally, induced pluripotent stem cell (iPS) technology holds promise for FA therapy (Table 2). Direct reprogramming of FA patient somatic cells into pluripotent stem cells, which retain the patient's unique genetic background, may provide a new source of autologous cells for transplant (115). The limitations of traditional gene therapy (e.g., difficulty in isolating sufficient HSCs) and the complications associated with bone marrow transplantation may be avoided by transplanting cDNA-corrected iPS cells to FA patients $(115,116)$. Several issues must be resolved before successful application to the clinic. As for all iPS approaches, use of proto-oncogenes and viral-mediated gene insertion increases the risk of oncogenesis. A safer and more sequence-specific method of gene introduction will be required. Also, the reprogramming efficiency of FA cells must be improved. Knockdown of the FA pathway leads to loss of self-renewal potency, which suggests that a functional FA pathway is required for induction and maintenance of pluripotency (115). However, a recent study demonstrated that functional complementation of the FA pathway restores the reprogramming efficiency of FA patient cells, providing a strategy in FA-specific iPS generation (117). Finally, successful induction of blood progenitors from iPS cells, capable of long-term hematopoietic reconstitution, at a scale compatible for transplantation in FA patients remains a challenge $(116,118)$. Despite these challenges, iPS technology offers the promise of generating tissue-compatible transplants for all FA patients.

\section{Acknowledgments}

We apologize for those whose work is not cited due to space limitations. We thank Alexandra Feinstein for critical reading of the manuscript. This work was supported by NIH grants R01DK43889, R37HL52725, and 2P01HL048546 to A.D. D'Andrea and by departmental startup funds to Y. Kee.

Address correspondence to: Younghoon Kee, University of South Florida, Department of Cell Biology, Microbiology, and Molecular Biology, 216 Bio-Science Facility Building, Tampa, Florida 33620, USA. Phone: 813.974.5352; Fax: 813.974.1614; E-mail: ykee@ usf.edu. Or to: Alan D. D’Andrea, Dana Farber Cancer Institute, Radiation Oncology, Mayer Bldg., Rm. 640, 450 Brookline Ave., Boston, Massachusetts 02215, USA. Phone: 617.632.2112; Fax: 617.632.5757; E-mail: alan_dandrea@dfci.harvard.edu.
1. D'Andrea AD. Susceptibility pathways in Fanconi's anemia and breast cancer. $N$ Engl J Med. 2010;362(20):1909-1919.

2. Auerbach AD. Fanconi anemia diagnosis and the diepoxybutane (DEB) test. Exp Hematol. 1993;21(6):731-733

3. van der Lelij P, Oostra AB, Rooimans MA, Joenje H, de Winter JP. Diagnostic overlap between Fanconi anemia and the Cohesinopathies: Roberts Syndrome and Warsaw Breakage Syndrome. Anemia. 2010;2010:565268.

4. Soulier J. Fanconi anemia. Hematology Am Soc Hematol Educ Program. 2011;2011:492-497.

5. Giampietro PF, Adler-Brecher B, Verlander PC, Pavlakis SG, Davis JG, Auerbach AD. The need for more accurate and timely diagnosis in Fanconi anemia: a report from the International Fanconi Anemia Registry. Pediatrics. 1993;91(6):1116-1120.

6. Giri N, Batista DL, Alter BP, Stratakis CA. Endocrine abnormalities in patients with Fanconi anemia. J Clin Endocrinol Metab. 2007;92(7):2624-2631.

7. Elder DA, et al. Abnormalities in glucose tolerance are common in children with fanconi anemia and associated with impaired insulin secretion. Pediatr Blood Cancer. 2008;51(2):256-260.

8. Wajnrajch MP, et al. Evaluation of growth and hormonal status in patients referred to the International Fanconi Anemia Registry. Pediatrics. 2001;107(4):744-754.

9. Rietschel P, et al. Assessment of growth hormone dynamics in human immunodeficiency virusrelated lipodystrophy. J Clin Endocrinol Metab. 2001;86(2):504-510.

10. Butturini A, Gale RP, Verlander PC, Adler-Brecher B, Gillio AP, Auerbach AD. Hematologic abnormalities in Fanconi anemia: an International Fanconi Anemia Registry study. Blood. 1994;84(5):1650-1655.

11. Kutler DI, et al. A 20-year perspective on the Inter- national Fanconi Anemia Registry (IFAR). Blood. 2003;101(4):1249-1256.

12. Quentin S, et al. Myelodysplasia and leukemia of Fanconi anemia are associated with a specific pattern of genomic abnormalities that includes cryptic RUNX1/ AML1 lesions. Blood. 2011;117(15):e161-170.

13. Meyer S, et al. Fanconi anemia (FA)-associated $3 q$ gains in leukemic transformation consistently target EVI1, but do not affect low TERC expression in FA. Blood. 2011;117(22):6047-6050.

14. Meyer S, et al. Amplification and translocation of 3 q26 with overexpression of EVI1 in Fanconi anemia-derived childhood acute myeloid leukemia with biallelic FANCD1/BRCA2 disruption. Genes Chromosomes Cancer. 2007;46(4):359-372.

15. Du W, Adam Z, Rani R, Zhang X, Pang Q. Oxidative stress in Fanconi anemia hematopoiesis and disease progression. Antioxid Redox Signal. 2008;10(11):1909-1921.

16. Taniguchi T, D'Andrea AD. Molecular pathogenesis of Fanconi anemia: recent progress. Blood. 2006;107(11):4223-4233.

17. Kutler DI, et al. High incidence of head and neck squamous cell carcinoma in patients with Fanconi anemia. Arch Otolaryngol Head Neck Surg. 2003;129(1):106-112.

18. Rosenberg PS, Greene MH, Alter BP. Cancer incidence in persons with Fanconi anemia. Blood. 2003;101(3):822-826.

19. Kutler DI, et al. Human papillomavirus DNA and p53 polymorphisms in squamous cell carcinomas from Fanconi anemia patients. J Natl Cancer Inst. 2003;95(22):1718-1721.

20. van Zeeburg HJ, Snijders PJ, Joenje H, Brakenhoff RH. Re: Human papillomavirus DNA and p53 polymorphisms in squamous cell carcinomas from Fanconi anemia patients. J Natl Cancer Inst. 2004;96(12):968.
21. van Zeeburg HJ, et al. Clinical and molecular characteristics of squamous cell carcinomas from Fanconi anemia patients. J Natl Cancer Inst. 2008;100(22):1649-1653.

22. Alter BP, et al. Fanconi's anaemia and pregnancy. BrJ Haematol. 1991;77(3):410-418.

23. Nabhan SK, et al. Fertility recovery and pregnancy after allogeneic hematopoietic stem cell transplantation in Fanconi anemia patients. Haematologica. 2010;95(10):1783-1787.

24. Parmar K, D’Andrea A, Niedernhofer LJ. Mouse models of Fanconi anemia. Mutat Res. 2009; 668(1-2):133-140.

25 . Koomen $\mathrm{M}$, et al. Reduced fertility and hypersensitivity to mitomycin C characterize Fancg/Xrcc9 null mice. Hum Mol Genet. 2002;11(3):273-281.

26. Kee Y, D'Andrea AD. Expanded roles of the Fanconi anemia pathway in preserving genomic stability. Genes Dev. 2010;24(16):1680-1694.

27. Howlett NG, et al. Biallelic inactivation of BRCA2 in Fanconi anemia. Science. 2002;297(5581):606-609.

28. Garcia-Higuera I, et al. Interaction of the Fanconi anemia proteins and BRCA 1 in a common pathway. Mol Cell. 2001;7(2):249-262.

29. Xia B, et al. Fanconi anemia is associated with a defect in the BRCA2 partner PALB2. Nat Genet. 2007;39(2):159-161.

30. Reid S, et al. Biallelic mutations in PALB2 cause Fanconi anemia subtype FA-N and predispose to childhood cancer. Nat Genet. 2007;39(2):162-164.

31. Levitus M, et al. The DNA helicase BRIP1 is defective in Fanconi anemia complementation group J. Nat Genet. 2005;37(9):934-935.

32. Litman R, et al. BACH1 is critical for homologous recombination and appears to be the Fanconi anemia gene product FANCJ. Cancer Cell. 2005;8(3):255-265.

33. Meindl A, et al. Germline mutations in breast 
and ovarian cancer pedigrees establish RAD51C as a human cancer susceptibility gene. Nat Genet. 2010;42(5):410-414.

34. Vaz F, et al. Mutation of the RAD51C gene in a Fanconi anemia-like disorder. Nat Genet. 2010; 42(5):406-409.

35. Myers K, et al. The clinical phenotype of children with Fanconi anemia caused by biallelic FANCD1/BRCA2 mutations. Pediatr Blood Cancer. 2012;58(3):462-465.

36. Guervilly JH, Mace-Aime G, Rosselli F. Loss of CHK1 function impedes DNA damage-induced FANCD2 monoubiquitination but normalizes the abnormal G2 arrest in Fanconi anemia. Hum Mol Genet. 2008;17(5):679-689.

37. Machida YJ, et al. UBE2T is the E2 in the Fanconi anemia pathway and undergoes negative autoregulation. Mol Cell. 2006;23(4):589-596.

38. Smogorzewska A, Matsuoka S, Vinciguerra P, McDonald ER. Identification of the FANCI protein, a monoubiquitinated FANCD2 paralog required for DNA repair. Cell. 2007;129(2):289-301.

39. Sims AE, Spiteri E, Sims RJ. FANCI is a second monoubiquitinated member of the Fanconi anemia pathway. Nat Struct Mol Biol. 2007;14(6):564-567.

40. Joo W, et al. Structure of the FANCI-FANCD2 complex: insights into the Fanconi anemia DNA repair pathway. Science. 2011;333(6040):312-316.

41. Liu T, Ghosal G, Yuan J, Chen J, Huang J. FAN1 acts with FANCI-FANCD2 to promote DNA interstrand cross-link repair. Science. 2010;329(5992):693-696

42. Smogorzewska A, et al. A genetic screen identifies FAN1, a Fanconi anemia-associated nuclease necessary for DNA interstrand crosslink repair. Mol Cell. 2010;39(1):36-47.

43. Kratz K, et al. Deficiency of FANCD2-associated nuclease KIAA1018/FAN1 sensitizes cells to interstrand crosslinking agents. Cell. 2010;142(1):77-88.

44. MacKay C, et al. Identification of KIAA1018/ FAN1, a DNA repair nuclease recruited to DNA damage by monoubiquitinated FANCD2. Cell. 2010;142(1):65-76.

45. Hussain S, et al. Direct interaction of FANCD2 with BRCA2 in DNA damage response pathways. Hum Mol Genet. 2004;13(12):1241-1248.

46. Wang X, Andreassen PR, D'Andrea AD. Functional interaction of monoubiquitinated FANCD2 and BRCA2/FANCD1 in chromatin. Mol Cell Biol. 2004;24(13):5850-5862.

47. Xia B, et al. Control of BRCA2 cellular and clinical functions by a nuclear partner, PALB2. Mol Cell. 2006;22(6):719-729.

48. Taniguchi T, Garcia-Higuera I, Andreassen PR, Gregory RC, Grompe M, D’Andrea AD. S-phasespecific interaction of the Fanconi anemia protein, FANCD2, with BRCA1 and RAD51. Blood. 2002;100(7):2414-2420.

49. Long DT, Raschle M, Joukov V, Walter JC. Mechanism of RAD51-dependent DNA interstrand crosslink repair. Science. 2011;333(6038):84-87.

50. Adamo A, et al. Preventing nonhomologous end joining suppresses DNA repair defects of Fanconi anemia. Mol Cell. 2010;39(1):25-35.

51. Pace P, Mosedale G, Hodskinson MR, Rosado IV, Sivasubramaniam M, Patel KJ. Ku70 corrupts DNA repair in the absence of the Fanconi anemia pathway. Science. 2010;329(5988):219-223.

52. Moldovan GL, D'Andrea AD. How the fanconi anemia pathway guards the genome. Annu Rev Genet. 2009;43:223-249.

53. Zhang XY, Langenick J, Traynor D, Babu MM, Kay RR, Patel KJ. Xpf and not the Fanconi anaemia proteins or Rev3 accounts for the extreme resistance to cisplatin in Dictyostelium discoideum. PLoS Genet. 2009;5(9):e1000645.

54. Prasher JM, et al. Reduced hematopoietic reserves in DNA interstrand crosslink repair-deficient
Ercc1 $^{-/-}$mice. EMBO J. 2005;24(4):861-871.

55. Niedzwiedz W, Mosedale G, Johnson M, Ong CY, Pace P, Patel KJ. The Fanconi anaemia gene FANCC promotes homologous recombination and errorprone DNA repair. Mol Cell. 2004;15(4):607-620.

56. Nojima K, et al. Multiple repair pathways mediate tolerance to chemotherapeutic cross-linking agents in vertebrate cells. Cancer Res. 2005; 65(24):11704-11711.

57. Knipscheer $\mathrm{P}$, et al. The Fanconi anemia pathway promotes replication-dependent DNA interstrand cross-link repair. Science. 2009; 326(5960):1698-1701.

58. Bhagwat N, et al. XPF-ERCC1 participates in the Fanconi anemia pathway of cross-link repair. Mol Cell Biol. 2009;29(24):6427-6437.

59. Kim Y, Lach FP, Desetty R, Hanenberg H, Auerbach AD, Smogorzewska A. Mutations of the SLX4 gene in Fanconi anemia. Nat Genet. 2011;43(2):142-146.

60 . Stoepker C, et al. SLX4, a coordinator of structurespecific endonucleases, is mutated in a new Fanconi anemia subtype. Nat Genet. 2011;43(2):138-141.

61. Crossan GP, et al. Disruption of mouse Slx4, a regulator of structure-specific nucleases, phenocopies Fanconi anemia. Nat Genet. 2011;43(2):147-152.

62. Yamamoto $\mathrm{KN}$, et al. Involvement of SLX4 in interstrand cross-link repair is regulated by the Fanconi anemia pathway. Proc Natl Acad Sci U S A. 2011;108(16):6492-6496

63. Ciccia A, et al. Identification of FAAP24, a Fanconi anemia core complex protein that interacts with FANCM. Mol Cell. 2007;25(3):331-343.

64. Ling C, et al. FAAP100 is essential for activation of the Fanconi anemia-associated DNA damage response pathway. EMBOJ. 2007;26(8):2104-2114.

65. Singh TR, et al. MHF1-MHF2, a histone-foldcontaining protein complex, participates in the Fanconi anemia pathway via FANCM. Mol Cell. 2010;37(6):879-886.

66. Leung JW, Wang Y, Fong KW, Huen MS, Li L, Chen J. Fanconi anemia (FA) binding protein FAAP20 stabilizes FA complementation group A (FANCA) and participates in interstrand cross-link repair. Proc Natl Acad Sci U S A. 2012;109(12):4491-4496.

67. Ali AM, et al. FAAP20: a novel ubiquitin-binding FA nuclear core complex protein required for functional integrity of the FA-BRCA DNA repair pathway. Blood. 2012;119(14):3285-3294.

68. Kim H, Yang K, Dejsuphong D, D'Andrea AD. Regulation of Rev1 by the Fanconi anemia core complex. Nat Struct Mol Biol. 2012;19(2):164-170.

69. Cohn MA, et al. A UAF1-containing multisubunit protein complex regulates the Fanconi anemia pathway. Mol Cell. 2007;28(5):786-797.

70. Kim JM, et al. Inactivation of murine Usp1 results in genomic instability and a Fanconi anemia phenotype. Dev Cell. 2009;16(2):314-320.

71. Shigechi T, et al. ATR-ATRIP Kinase Complex Triggers Activation of the Fanconi Anemia DNA Repair Pathway. Cancer Res. 2012;72(5):1149-1156.

72. Collis SJ, Barber LJ, Clark AJ, Martin JS, Ward JD, Boulton SJ. HCLK2 is essential for the mammalian S-phase checkpoint and impacts on Chk1 stability. Nat Cell Biol. 2007;9(4):391-401.

73. Huang M, et al. Human MutS and FANCM complexes function as redundant DNA damage sensors in the Fanconi Anemia pathway. DNA Repair (Amst). 2011;10(12):1203-1212.

74. Deans AJ, West SC. FANCM connects the genome instability disorders Bloom's Syndrome and Fanconi Anemia. Mol Cell. 2009;36(6):943-953.

75. Wang W. Emergence of a DNA-damage response network consisting of Fanconi anaemia and BRCA proteins. Nat Rev Genet. 2007;8(10):735-748.

76. Porfirio B, et al. Fragile sites and chromosome instability: the distribution of breaks induced by cis-diamine-dichloro-platinum (II) in Fanconi anemia lymphocyte cultures. Hum Genet.
1991;86(3):256-260.

77. Fundia A, Gorla N, Larripa I. Spontaneous chromosome aberrations in Fanconi's anemia patients are located at fragile sites and acute myeloid leukemia breakpoints. Hereditas. 1994;120(1):47-50.

78. Vinciguerra P, Godinho SA, Parmar K, Pellman D, D'Andrea AD. Cytokinesis failure occurs in Fanconi anemia pathway-deficient murine and human bone marrow hematopoietic cells. J Clin Invest. 2010;120(11):3834-3842.

79. Naim V, Rosselli F. The FANC pathway and BLM collaborate during mitosis to prevent micro-nucleation and chromosome abnormalities. Nat Cell Biol. 2009;11(6):761-768.

80. Chan KL, Palmai-Pallag T, Ying S, Hickson ID. Replication stress induces sister-chromatid bridging at fragile site loci in mitosis. Nat Cell Biol. 2009;11(6):753-760.

81. Alter BP. Diagnosis, genetics, and management of inherited bone marrow failure syndromes. Hematology Am Soc Hematol Educ Program. 2007;2007:29-39.

82. Velazquez I, Alter BP. Androgens and liver tumors: Fanconi's anemia and non-Fanconi's conditions. Am J Hematol. 2004;77(3):257-267.

83. Scheckenbach K, et al. Treatment of the bone marrow failure in Fanconi anemia patients with danazol. Blood Cells Mol Dis. 2012;48(2):128-131.

84. Alter BP, Caruso JP, Drachtman RA, Uchida T, Velagaleti GV, Elghetany MT. Fanconi anemia: myelodysplasia as a predictor of outcome. Cancer Genet Cytogenet. 2000;117(2):125-131.

85. MacMillan ML, Wagner JE. Haematopoeitic cell transplantation for Fanconi anaemia - when and how? Br J Haematol. 2010;149(1):14-21.

86. Zanis-Neto J, et al. Low-dose cyclophosphamide conditioning for haematopoietic cell transplantation from HLA-matched related donors in patients with Fanconi anaemia. Br J Haematol. 2005;130(1):99-106.

87. Pasquini R, et al. HLA-matched sibling hematopoietic stem cell transplantation for fanconi anemia: comparison of irradiation and nonirradiation containing conditioning regimens. Biol Blood Marrow Transplant. 2008;14(10):1141-1147.

88. Shimada A, et al. Excellent outcome of allogeneic bone marrow transplantation for Fanconi anemia using fludarabine-based reduced-intensity conditioning regimen. Int J Hematol. 2012;95(6):675-679.

89. MacMillan ML, et al. Haematopoietic cell transplantation in patients with Fanconi anaemia using alternate donors: results of a total body irradiation dose escalation trial. Br J Haematol. 2000;109(1):121-129.

90. Verlinsky Y, Rechitsky S, Schoolcraft W, Strom C, Kuliev A. Preimplantation diagnosis for Fanconi anemia combined with HLA matching. JAMA. 2001;285(24):3130-3133.

91. Gluckman E, et al. Bone marrow transplantation for Fanconi anemia. Blood. 1995;86(7):2856-2862.

92. Watts KL, Delaney C, Humphries RK, Bernstein ID, Kiem HP. Combination of HOXB4 and Delta1 ligand improves expansion of cord blood cells. Blood. 2010;116(26):5859-5866.

93. Becker PS, et al. Preclinical correction of human Fanconi anemia complementation group A bone marrow cells using a safety-modified lentiviral vector. Gene Ther. 2010;17(10):1244-1252.

94. Eyal O, Blum S, Mueller R, Smith FO, Rose SR. Improved growth velocity during thyroid hormone therapy in children with Fanconi anemia and borderline thyroid function. Pediatr Blood Cancer. 2008;51(5):652-656.

95. Dupuis-Girod S, Gluckman E, Souberbielle JC, Brauner R. Growth hormone deficiency caused by pituitary stalk interruption in Fanconi's anemia. JPediatr. 2001;138(1):129-133.

96. Alter BP, Greene MH, Velazquez I, Rosenberg PS. Cancer in Fanconi anemia. Blood. 2003;101(5):2072. 
97. Frappart PO, Lee Y, Lamont J, McKinnon PJ. BRCA2 is required for neurogenesis and suppression of medulloblastoma. EMBOJ. 2007;26(11):2732-2742.

98. Hirsch B, et al. Association of biallelic BRCA2 FANCD1 mutations with spontaneous chromosomal instability and solid tumors of childhood. Blood. 2004;103(7):2554-2559.

99. Park JW, et al. Deficiencies in the Fanconi anemia DNA damage response pathway increase sensitivity to HPV-associated head and neck cancer. Cancer Res. 2010;70(23):9959-9968.

100. Rosado IV, Langevin F, Crossan GP, Takata M, Patel $\mathrm{KJ}$. Formaldehyde catabolism is essential in cells deficient for the Fanconi anemia DNA-repair pathway. Nat Struct Mol Biol. 2011;18(12):1432-1434.

101. Ridpath JR, et al. Cells deficient in the FANC/BRCA pathway are hypersensitive to plasma levels of formaldehyde. Cancer Res. 2007;67(23):11117-11122.

102.Ito K, et al. Regulation of oxidative stress by ATM is required for self-renewal of haematopoietic stem cells. Nature. 2004;431(7011):997-1002.

103. Nijnik A, et al. DNA repair is limiting for haematopoietic stem cells during ageing. Nature. 2007;447(7145):686-690.

104.Zhang QS, et al. Fancd2-/- mice have hematopoi- etic defects that can be partially corrected by resveratrol. Blood. 2010;116(24):5140-5148.

105.Zhang QS, et al. Tempol protects against oxidative damage and delays epithelial tumor onset in Fanconi anemia mice. Cancer Res. 2008;68(5):1601-1608.

106. Mankad A, et al. Natural gene therapy in monozygotic twins with Fanconi anemia. Blood. 2006; 107(8):3084-3090.

107. Waisfisz Q, et al. Spontaneous functional correction of homozygous fanconi anaemia alleles reveals novel mechanistic basis for reverse mosaicism. Nat Genet. 1999;22(4):379-383.

108. Gross M, et al. Reverse mosaicism in Fanconi anemia: natural gene therapy via molecular self-correction. Cytogenet Genome Res. 2002;98(2-3):126-135.

109. Soulier J, et al. Detection of somatic mosaicism and classification of Fanconi anemia patients by analysis of the FA/BRCA pathway. Blood. 2005; 105(3):1329-1336.

110. Gostynski A, Deviaene FC, Pasmooij AM, Pas HH, Jonkman MF. Adhesive stripping to remove epidermis in junctional epidermolysis bullosa for revertant cell therapy. BrJ Dermatol. 2009;161(2):444-447.

111.Lai-Cheong JE, McGrath JA, Uitto J. Revertant mosaicism in skin: natural gene therapy. Trends Mol
Med. 2011;17(3):140-148

112.Gregory JJ, et al. Somatic mosaicism in Fanconi anemia: evidence of genotypic reversion in lymphohematopoietic stem cells. Proc Natl Acad Sci U S A. 2001;98(5):2532-2537.

113. Ceccaldi R, et al. Spontaneous abrogation of the GDNA damage checkpoint has clinical benefits but promotes leukemogenesis in Fanconi anemia patients. J Clin Invest. 2011;121(1):184-194.

114.Narla A, Vlachos A, Nathan DG. Diamond Blackfan anemia treatment: past, present, and future. Semin Hematol. 2011;48(2):117-123.

115. Raya A, et al. Disease-corrected haematopoietic progenitors from Fanconi anaemia induced pluripotent stem cells. Nature. 2009;460(7251):53-59.

116. MacMillan ML, Hughes MR, Agarwal S, Daley GQ. Cellular therapy for fanconi anemia: the past, present, and future. Biol Blood Marrow Transplant. 2011; 17(1 suppl):S109-S114.

117. Müller LU, et al. Overcoming reprogramming resistance of Fanconi anemia cells. Blood. 2012; 119(23):5449-5457.

118. Robinton DA, Daley GQ. The promise of induced pluripotent stem cells in research and therapy. Nature. 2012;481(7381):295-305 